\title{
Strand-specific detection of overlapping transcripts via purification involving denaturation of biotinylated cDNA
}

\author{
Faizan Uddin ${ }^{1}$ \& Madhulika Srivastava*,1 (iD \\ ${ }^{1}$ Epigenetics Research Laboratory, National Institute of Immunology, Aruna Asaf Ali Marg, New Delhi, 110067, India; *Author for correspondence: madhu@nii.ac.in
}

BioTechniques 69: 141-147 (August 2020) 10.2144/btn-2020-0008

First draft submitted: 17 January 2020; Accepted for publication: 31 March 2020; Published online: 6 May 2020

\section{ABSTRACT}

Reverse transcription-PCR (RT-PCR) is the most widely employed technique for gene expression analysis owing to its high sensitivity, easy reproducibility and fast output. It has been conceived that priming RT reactions with gene-specific primers generates cDNA only from the specific RNA. However, several reports have revealed that cDNA is synthesized even without addition of exogenous primers in RT reactions. Owing to such self-priming activity, the signals from specific strands cannot be accurately detected and can confound the expression analysis, especially in context of overlapping bidirectional transcripts. Here, we demonstrate that purification of biotin-tagged cDNA in conjunction with alkaline denaturation can obviate the problem of background priming and enable accurate strand-specific detection of overlapping transcripts.

\section{METHOD SUMMARY}

cDNAs synthesized with biotin-tagged primers were bound to streptavidin magnetic beads followed by alkaline denaturation to disrupt cDNAcDNA duplex, potentially formed by sense and antisense overlapping cDNAs. Untagged cDNAs synthesized owing to self-priming were subsequently washed out, and biotin-tagged cDNA of interest was selectively enriched.

\section{KEYWORDS:}

alkaline denaturation $\bullet$ biotinylated primers $\bullet$ detection of overlapping transcripts $\bullet$ self-priming $\bullet$ strand-specific reverse transcriptionPCR • streptavidin magnetic beads

One of the most widely used molecular techniques for gene expression analysis is reverse transcription-PCR (RT-PCR), which bestows several advantages in being highly reproducible and sensitive, requiring less sample RNA and permitting simultaneous and rapid analysis of multiple genes. A typical RT reaction utilizes an oligonucleotide DNA primer, which generates a single-stranded cDNA using the RNA as a template. It is generally assumed that priming RT with gene-specific primer (GSP) for a particular transcript would generate cDNA only from that desired RNA template and guarantee the specificity of signal in subsequent amplification by PCR. However, the conventional notion regarding absolute requirement of the exogenous primers during RT has been refuted by several reports involving detection of plant [1,2], animal [3-5] and human viruses [6,7] as well as expression of genes in eukaryotes [6-9], in which it was observed that cDNAs can be synthesized even without adding exogenous primers to the RT reaction. Generation of such nonspecific cDNAs through a primer independent mechanism has been linked to self-priming activity of the RNA template by its own 3' end [6] and previously termed as background priming $[4,8]$.

The effect of background priming has been largely ignored in routine gene expression analysis of unidirectional nonoverlapping transcripts, as their final detection by PCR remains unaltered in the presence of self-primed cDNAs generated from transcripts at other loci. Conversely, the presence of self-primed cDNAs would severely impair the analysis of overlapping transcripts when both strands are co-expressed. In such a scenario, the signal from specifically primed cDNA would be impossible to differentiate from the nonspecific cDNA arising, owing to background priming. This problem has been best described in studies involving RNA viruses such as Dengue virus, West Nile virus, Norovirus and Coxsackievirus B5 [6,10-12]. In these viruses, the expression of an intermediate transcript of negative orientation has been used as a marker for active viral replication and the underlying progressing pathogenesis. However, owing to generation of the self-primed cDNA from the positive-strand RNA, constituting the genome of the virus, it was unfeasible to accurately measure the levels of the negative-stranded intermediate form of RNA viruses in the infected cells. Consequently, RT-PCR could not give reliable estimates of persistent viral replication, which in some cases has been linked with chronic diseases, including dilated cardiomyopathy caused by Coxsackievirus B5 [12]. The contribution of cDNA originating from the incorrect negative strand can be substantially high, ranging from 30 to $50 \%$, depending on RNA secondary structure and reaction conditions during RT-PCR [6,7,10]. 


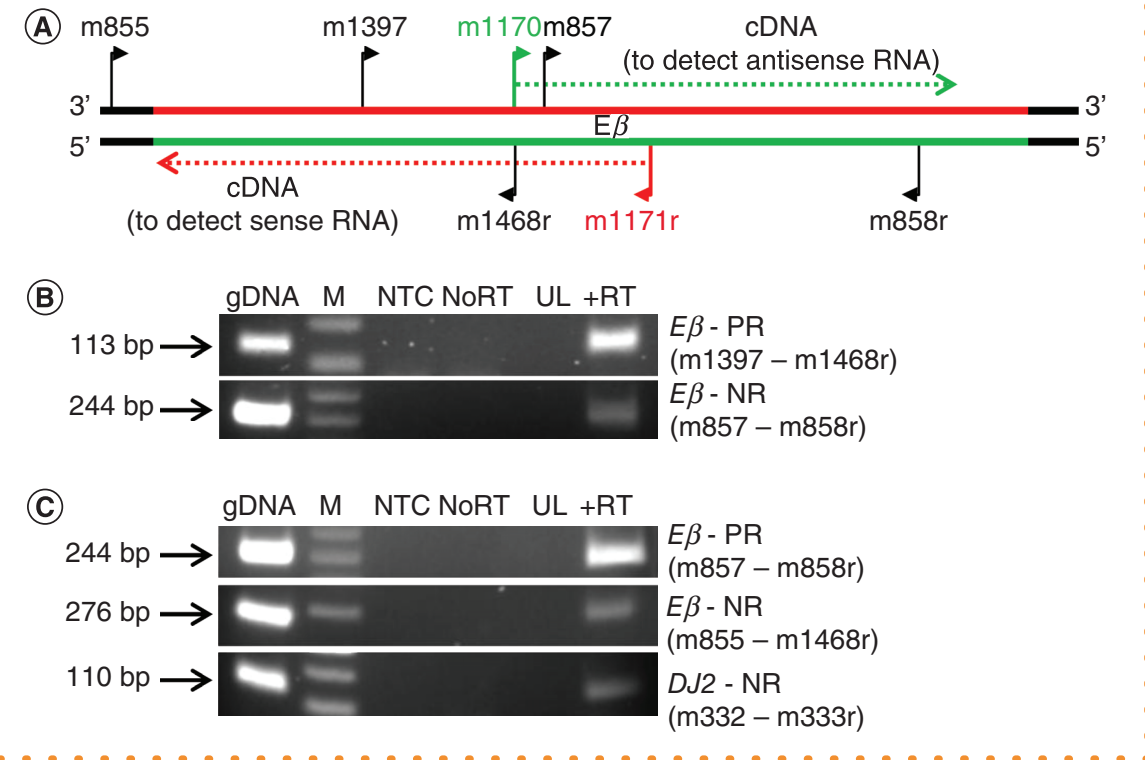

Figure 1. Transcript analysis of enhancer $E \beta$ by RT-PCR. (A) Position of primers used for reverse transcription and PCR. Red and green solid lines represent sense and antisense strand of $E \beta$ span, respectively. (B) PCR amplification of cDNA synthesized using GSP m1171r to detect sense transcripts. (C) PCR amplification of cDNA synthesized using GSP m1170 to detect antisense transcripts. Primers used in PCR amplification are mentioned alongside the amplicons detected. PR (region expected to be amplified) and NR (region not expected to be amplified) were ascribed based on location of GSP used for CDNA synthesis.

M: DNA molecular weight marker; NTC: No template control; UL: Unused lane.

(A)

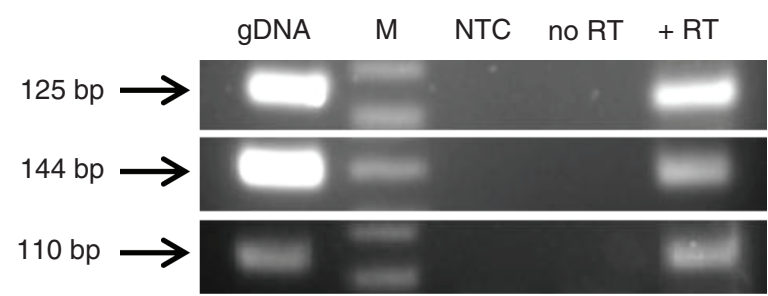

GAPDH - PR

$(\mathrm{m} 2091-\mathrm{m} 2092 \mathrm{r})$

DJ1 - NR

$(\mathrm{m} 703-\mathrm{m} 704 \mathrm{r})$

Thy1.2 - NR

(m558- m559r)

(B)
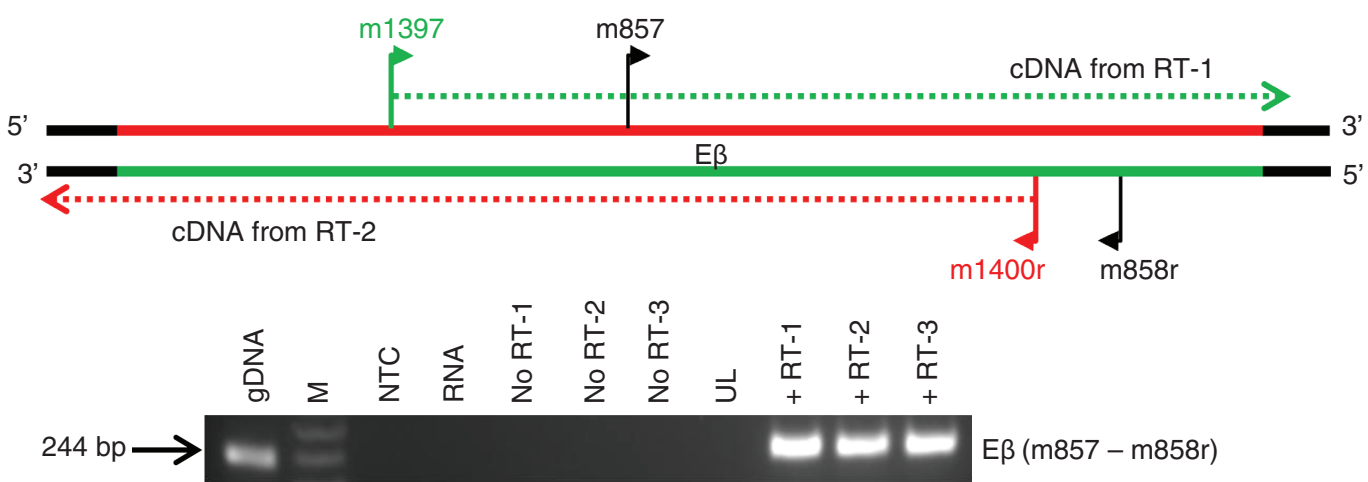

PR NR NR

Figure 2. Detection of self-primed cDNA at various loci. (A) CDNA synthesized with GAPDH-specific primer showing PCR amplification to detect GAPDH, DJ1 and Thy1.2 transcripts. (B) Top: Position of primers used for cDNA synthesis and subsequent PCR amplification at enhancer E $\beta$. RT-1 and RT-2 were primed with GSP m1397 and m1400r, respectively. RT-3 was carried out without any exogenous primers. Red and green solid lines represent sense and antisense strand of $E \beta$ span, respectively. Bottom: PCR amplification of cDNA from RT-1, 2 and 3. PR (region expected to be amplified) and NR (region not expected to be amplified) were ascribed based on location of GSP used for CDNA synthesis. M: DNA molecular weight marker; NTC: No template control; RNA: RNA after DNase-I treatment; UL: Unused lane; RT: Reverse transcriptase. 


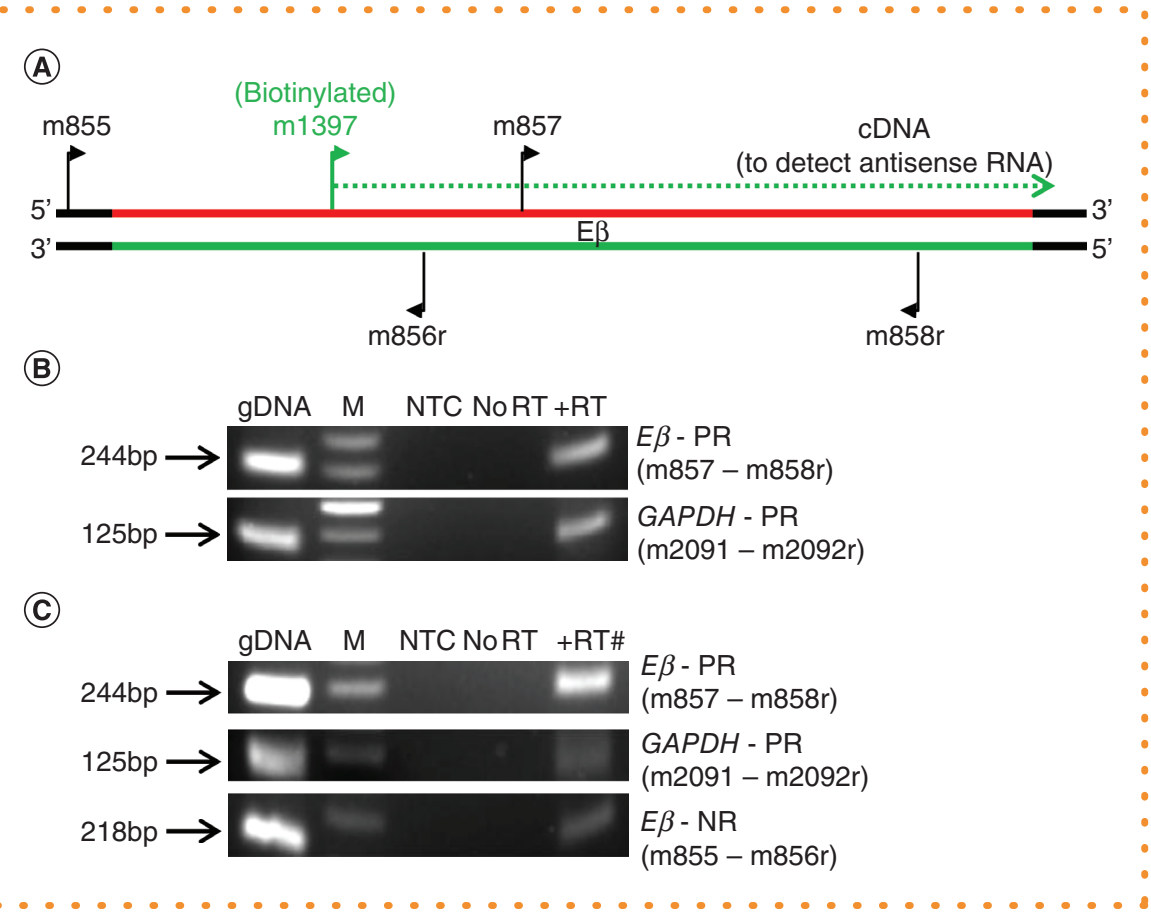

Figure 3. Analysis of biotinylated cDNA after enrichment via streptavidin magnetic beads as described by Boncristiani et al. [12]. (A) Position of $E \beta$-specific primers used for reverse transcription and PCR. Reverse transcription was primed with biotinylated GSP m1397 and GAPDH-specific biotinylated primer $\mathrm{m} 2092 \mathrm{r}$. Red and green bold lines represent sense and antisense strand of $E \beta$ span, respectively. (B) Top: PCR amplification of cDNA before selective enrichment of biotinylated cDNA. Bottom: PCR amplification of enriched biotinylated cDNA. Primers used in PCR amplification are mentioned alongside for the amplicons detected. PR (region expected to be amplified) and NR (region not expected to be amplified) were ascribed based on location of GSP used for CDNA synthesis.

"Use of Enriched biotinylated cDNA for PCR.

M: DNA molecular weight marker; NTC: No template control; RT: Reverse transcriptase.

The problem associated with self-primed cDNA is further highlighted in the context of eukaryotic cellular mRNAs by a study involving isoforms of myosin heavy chain motor protein, encoded from MYH6 and MYH7 genes in rat muscle cells [9]. In induced hypothyroid state, both the sense and antisense transcripts from the MYH7 gene were found to be transcribed simultaneously; however, their individual levels could not be precisely quantitated by RT-PCR primed with strand-specific primers. Moreover, self-primed cDNA was synthesized in RT reactions without addition of exogenous primers. Prevalent overlapping bidirectional transcription was also reported in a wide range of organisms to generate functional natural antisense RNA pairs that are co-expressed at differential levels [13,14].

To circumvent the problem of background priming in RT-PCR, a multitude of approaches have been adopted at various stages, such as additional processing of sample RNA, variations at the level of CDNA synthesis and PCR amplification after RT [1-3,5-12]. These strategies include elimination of free nucleic acid contaminants from the RNA sample that can act as endogenous primers, RNase- $\mathrm{H}$ mediated digestion of nontarget RNA strand, performing RT at a higher extension temperature, addition of DMSO to RT reaction, use of a thermostable reverse transcriptase, using reverse transcriptase with RNase-H activity and using one-step RT-PCR kits. Furthermore, the incorporation of additional tags in RT primers and subsequent detection of tagged cDNAs, modifying the sequence of primer-specific CDNA, digestion of unused RT primers with Exonuclease-I and purification of CDNA synthesized with biotinylated primers have also been tested in several methods. Although these strategies when applied alone did not improve the strand-specific detection of cDNAs [1,3,68,12], few combinatorial approaches have shown a varying degree of success [2,5,9-11].

Active enhancer domains have been reported to be transcribed, bidirectionally or unidirectionally, and produce transcripts that exhibit precise tissue and developmental stage- specific activity $[15,16]$. Enhancer $E \beta$ is an important regulatory region of murine TCR $\beta$ locus (chromosome 6) [17-19]. To understand its role in T-cell development, we investigated transcription at the $E \beta$ enhancer. We detected transcripts from $E \beta$ in CDNA primed with either GSPs m1171r or m1170 during RT-PCR at $50^{\circ} \mathrm{C}$. Unexpectedly, both cDNAs tested positive for amplicons spanning regions outside the GSP used for CDNA synthesis (Figure 1). We designate such a region, where amplification was not expected, as negative region (NR) as opposed to a positive region (PR) where amplification was expected. Further, we observed that cDNA was also amplified with primers in the $D J C \beta 2$ region (DJ2) located $12 \mathrm{~kb}$ upstream to $E \beta$ even though primers for $D J 2$ were not added in the RT reaction (Figure 1C).

These observations prompted us to test few other genes such as $D J 1$ (in the $D J C \beta 1$ region located $21 \mathrm{~kb}$ upstream to $E \beta$ ) and Thy1.2 (chromosome 9). In an RT primed only with a GAPDH-specific primer, amplification was observed for both DJ1 and Thy1.2 along with 
(A)

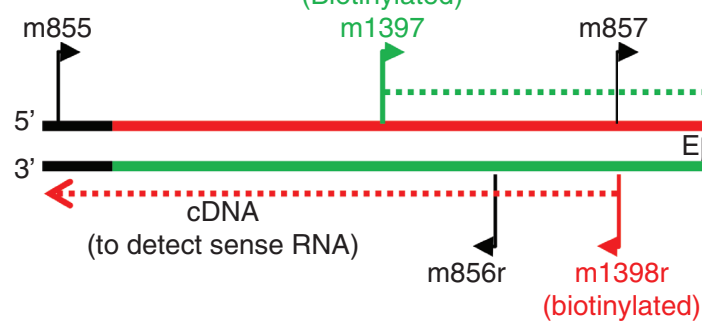

cDNA

(to detect antisense RNA)

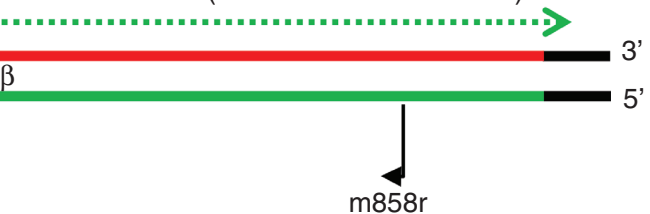

(B)
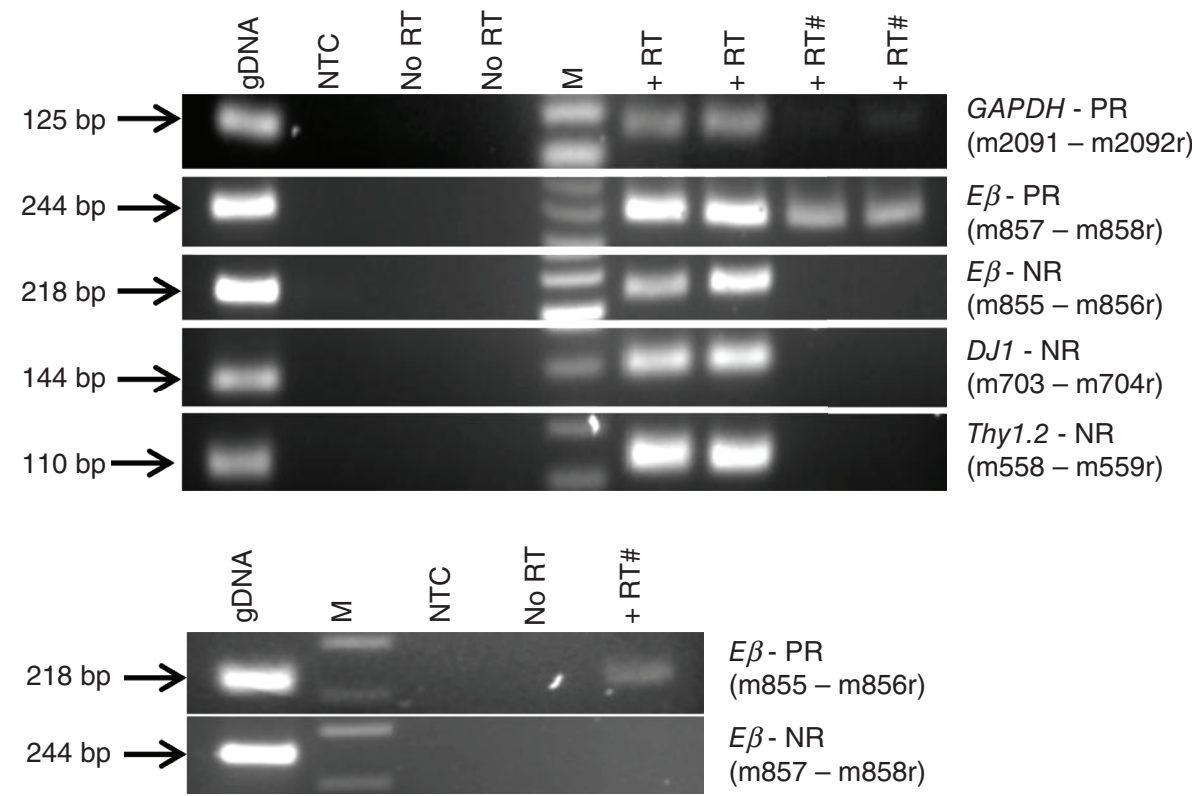

$E \beta-\mathrm{PR}$

$(\mathrm{m} 855-\mathrm{m} 856 \mathrm{r})$

$E \beta-\mathrm{NR}$

$(\mathrm{m} 857-\mathrm{m} 858 \mathrm{r})$

Figure 4. Strand-specific detection of $E \beta$ transcripts after selective enrichment of denatured biotinylated cDNA using modified strategy. (A) Position of $E \beta$-specific primers used for reverse transcription and PCR. Reverse transcription was primed with biotinylated GSPs $\mathrm{m} 1397$ and $\mathrm{m} 1398 \mathrm{r}$ to detect antisense and sense transcripts, respectively. Red and green solid lines represent sense and antisense strand of $E \beta$ span, respectively. (B) PCR amplification of cDNA primed with biotinylated GSP m1397 before and after selective enrichment of biotinylated cDNA to detect antisense transcripts. GAPDH-specific biotinylated primer $\mathrm{m} 2092 \mathrm{r}$ was added in the RT as positive control. (C) PCR amplification of cDNA primed with biotinylated GSP m1398r after selective enrichment of biotinylated cDNA to detect sense transcripts. Primers used in PCR amplification are mentioned alongside for the amplicons detected. PR (region expected to be amplified) and NR (region not expected to be amplified) were ascribed based on location of GSP used for cDNA synthesis.

\#Use of enriched biotinylated cDNA for PCR.

GSP: Gene-specific primer; M: DNA molecular weight marker; NTC: No template control; RT: Reverse transcriptase.

GAPDH (Figure 2A). Next, we compared GSP-primed cDNAs for each strand of $E \beta$ with a cDNA synthesized without addition of any exogenous primers (RT-1 with GSP m1397; RT-2 with GSP m1400r and RT-3 without primers) and performed RT at a higher temperature of $55^{\circ}$ C. However, all three CDNAs produced robust amplification in PCR, in which only RT-1 was expected to give amplification (Figure 2B). No amplification occurred in DNase I-treated RNA that was used for these RT reactions, therefore, ruling out any contamination of gDNA in the sample RNA. This led us to infer that RT-PCR cannot be used for strand-specific detection of overlapping bidirectional transcripts arising at $E \beta$. Also, an increased temperature of $55^{\circ} \mathrm{C}$ during RT was inadequate to eliminate background priming.

To overcome the problem of self-priming in RT-PCR, Boncristiani et al. demonstrated the effectiveness of streptavidin magnetic beads to bind biotinylated cDNA primed with biotinylated GSPs [12]. The methodology was used to enrich and detect biotinylated cDNA synthesized from an in vitro transcribed RNA, representing a single strand. Because this strategy is simple and straightforward, we attempted strand-specific detection of transcripts at $E \beta$ by enriching biotinylated cDNA for the specified transcript. Accordingly, cDNA for $E \beta$ antisense transcript was synthesized at $55^{\circ} \mathrm{C}$ using biotinylated $\mathrm{m} 1397$ and a biotinylated GSP for GAPDH. Biotinylated cDNAs were enriched using streptavidin magnetic beads and tested for amplification at PR and NR within $E \beta$. However, enriched cDNA was amplified from both regions and suggested that biotinylated cDNA could not be enriched with strand-specific selectivity (Figure 3). 


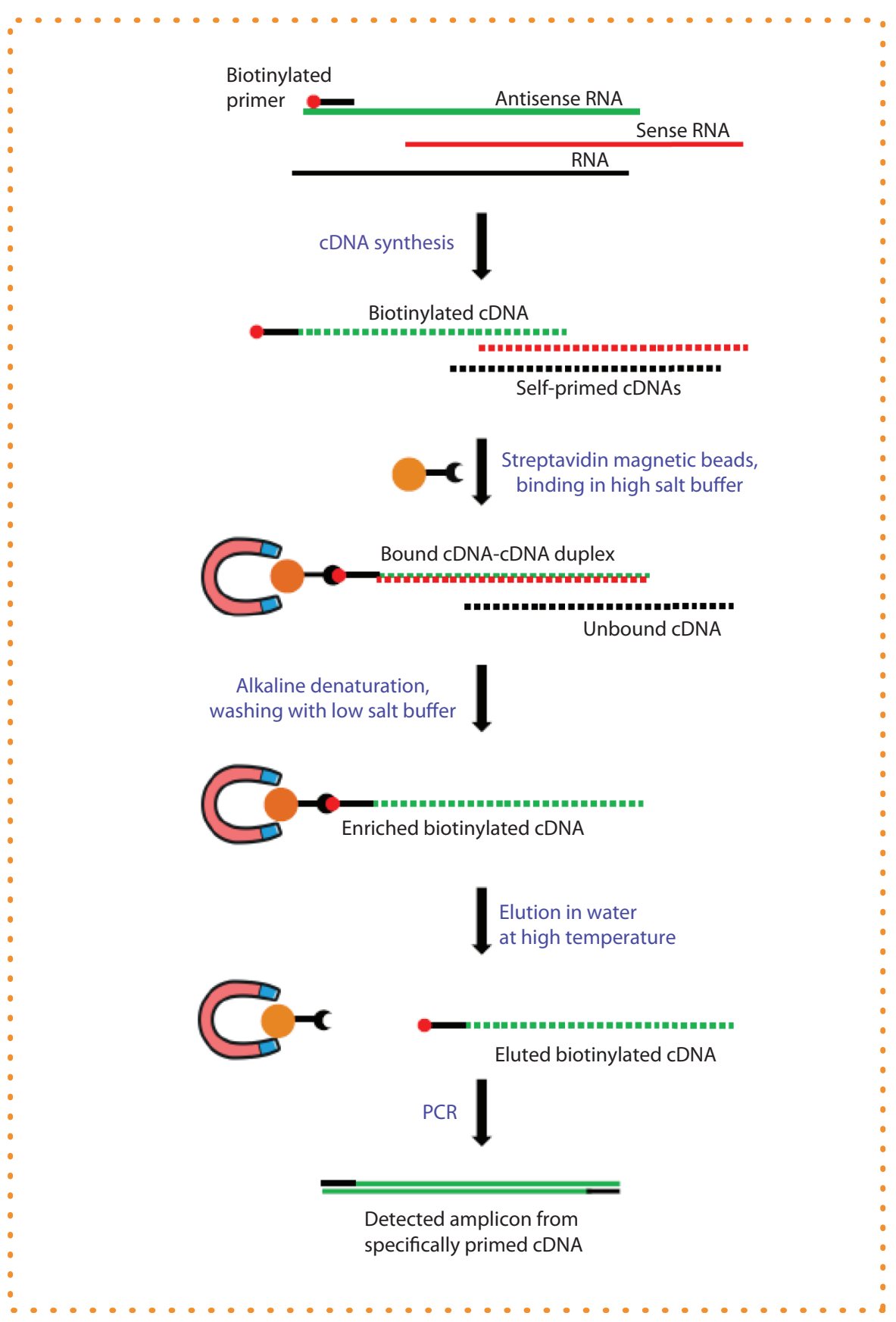

Figure 5. Protocol outline [20].

We hypothesized that a simple biotinylated cDNA enrichment assay [12] can perform well only when cDNA from the opposite overlapping strand is completely absent. In a situation when overlapping transcripts from both strands are co-expressed, as in cellular RNA samples, despite the use of a single biotinylated primer, cDNAs would be synthesized by appropriate priming as well as background priming. The resulting sense and antisense cDNAs can potentially form a cDNA-cDNA duplex. Consequently, the streptavidin magnetic beads can enrich the biotinylated cDNA that exist in single strands as well as cDNA-cDNA duplex form. This would foil the strandspecific detection of biotinylated cDNA during PCR. Here, we report a refinement of a previously published protocol [12] for specific enrichment of biotinylated cDNA by incorporation of an alkaline denaturation step to disrupt the cDNA-cDNA duplex to ensure removal of the paired self-primed cDNA.

Purification of cDNA primed with biotinylated m1397 using an in vitro transcribed antisense RNA as a template yielded enriched cDNA. Although the yield was low as ascertained by qPCR (Supplementary Figure 1), it was quite consistent and the purified cDNA was easily detectable. To test the efficiency of our protocol in detecting only specifically primed cDNA in total cellular RNA, RT reaction was 
primed using biotinylated GSPs for GAPDH and $E \beta$ antisense transcripts. Biotinylated cDNA, both before and after enrichment, was tested by PCR at the NR and PR of $E \beta$ along with DJ1 and Thy1.2 as additional NR. As expected, cDNA was amplified in all the tested regions before enrichment. However, after selective enrichment of denatured biotinylated cDNA specifically primed for GAPDH and $E \beta$ antisense transcripts were amplified at their respective PR by PCR (Figure 4A \& B). Both for E $\beta$-PR and GAPDH, there was a decrease in signal strength. This could arise owing to the combined effect of purification related loss as well as effective removal of the incorrect strand-based cDNA. Similar results were obtained by selective enrichment of biotinylated cDNA for $E \beta$ sense transcript (Figure $4 A \&$ C).

Importantly, no amplification was observed in any NR (Figure 4B \& C), indicating that our optimized protocol conferred enhanced accuracy in strand-specific detection of overlapping transcripts. Our optimized protocol [20] is outlined in Figure 5, and Supplementary Table 1 has the sequences of primers for reverse transcription and PCR used in this study.

This modified strategy is pertinent for expression analysis of bidirectional overlapping transcripts, particularly noncoding RNAs, many of which are co-expressed at very low levels. In such a scenario, background priming would not only interfere in accurate assessment of a particular transcript but also hinder correct estimation of the efficiency of knockdown during RNAi experiments aimed to deduce functionality of specific transcripts. In view of this, our modified protocol involving denaturation of cDNA-cDNA duplexes during enrichment of biotinylated cDNA can eliminate false positives in the RT-PCR arising due to background priming.

\section{Supplementary data}

To view the supplementary data that accompany this paper please visit the journal website at: www.futurescience.com/doi/suppl/10.2144/btn-2020-0008

\section{Author contributions}

F Uddin and M Srivastava designed the experiments. F Uddin performed the experiments. F Uddin and M Srivastava analyzed the data. F Uddin prepared the manuscript. M Srivastava revised the manuscript.

\section{Acknowledgments}

The authors would like to thank M Yadav for her assistance in editing the manuscript.

\section{Financial \& competing interests disclosure}

The study was supported by a grant (EMR/2016/006230) received from the Science and Energy Research Board, Department of Science and Technology, Government of India. The authors have no other relevant affiliations or financial involvement with any organization or entity with a financial interest in or financial conflict with the subject matter or materials discussed in the manuscript apart from those disclosed.

No writing assistance was utilized in the production of this manuscript.

\section{Ethical conduct of research}

All the experiments using animals were conducted in accordance with the Institutional Animal Ethics Committee of National Institute of Immunology.

\section{Open access}

This work is licensed under the Attribution-NonCommercial-NoDerivatives 4.0 Unported License. To view a copy of this license, visit http://creativecommons.org/licenses/by-nc-nd/4.0/

\section{References}

Papers of special note have been highlighted as: $\bullet$ of interest

1 Zhang C, Wu HN, Zhang YQ, Shen JG LW. Caracterization of a border disease virus isolate originating from Slovakia. Acta Virol. 59(1), 92-97 (2015).

2 Strydom E, Pietersen G. Development of a strand-specific RT-PCR to detect the positive sense replicative strand of Soybean blotchy mosaic virus. J. Virol. Methods 259(May), 39-44 (2018).

3 Haist K, Ziegler C, Botten J. Strand-specific quantitative reverse transcription-polymerase chain reaction assay for measurement of arenavirus genomic and antigenomic RNAs. PLoS ONE 10(5), 1-21 (2015).

4 Freeh B, Peterhans E. RT-PCR: "background priming” during reverse transcription. Nucleic Acids Res. 22(20), 4342-4343 (1994).

5 Tercero B, Terasaki K, Nakagawa K, Narayanan K, Makino S. A strand-specific real-time quantitative RT-PCR assay for distinguishing the genomic and antigenomic RNAs of Rift Valley fever phlebovirus. J. Virol. Methods 272(July), 113701 (2019).

6 Tuiskunen A, Leparc-Goffart I, Boubis L et al. Self-priming of reverse transcriptase impairs strand-specific detection of dengue virus RNA. J. Gen. Virol. 91(4), 1019-1027 (2010).

7 Feng L, Lintula S, Ho TH et al. Technique for strand-specific gene-expression analysis and monitoring of primer-independent cDNA synthesis in reverse transcription. BioTechniques 52 (4), 263-270 (2012)

- This study evaluated background priming in RT-PCR at various elongation temperatures and highlighted the magnitude of self-priming activity in a given RT reaction.

8 Yuan C, Liu Y, Yang M, Liao DJ. New methods as alternative or corrective measures for the pitfalls and artifacts of reverse transcription and polymerase chain reactions (RT-PCR) in cloning chimeric or antisense-accompanied RNA. RNA Biol. 10(6), 950-960 (2013).

9 Haddad F, Qin AX, Giger JM, Guo H, Baldwin KM. Potential pitfalls in the accuracy of analysis of natural sense-antisense RNA pairs by reverse transcription-PCR. BMC Biotechnol. 7, 1-14 (2007).

- This study reported the influence of background priming in the quantitation of natural sense-antisense RNA pairs in mammalian transcriptome. 
10 Lim SM, Koraka P, Osterhaus ADME, Martina BEE. Development of a strand-specific real-time qRT-PCR for the accurate detection and quantitation of West Nile virus RNA. J. Virol. Methods 194(1-2), 146-153 (2013).

- In this study, utility of various RT enzymes was evaluated in terms of degree of false-priming activity.

11 Vashist S, Urena L, Goodfellow I. Development of a strand specific real-time RT-qPCR assay for the detection and quantitation of murine norovirus RNA. J. Virol. Methods 184(1-2), 69-76 (2012).

12 Boncristiani HF, Rossi RD, Criado MF, Furtado FM, Arruda E. Magnetic purification of biotinylated cDNA removes false priming and ensures strand-specificity of RT-PCR for enteroviral RNAs. J. Virol. Methods 161(1), 147-153 (2009).

- This article demonstrated the utility of streptavidin magnetic beads to capture biotinylated cDNA for improving the detection of specifically primed cDNA.

13 Rosikiewicz W, Makatowska I. Biological functions of natural antisense transcripts. Acta Biochim. Pol. 63(4), 665-673 (2016).

14 Wanowska E, Kubiak MR, Rosikiewicz W, Makatowska I, Szcześniak MW. Natural antisense transcripts in diseases: from modes of action to targeted therapies. Wiley Interdiscip. Rev. RNA 9(2), 1-16 (2018).

- This review provides a comprehensive overview of the pervasive overlapping bidirectional transcription in the eukaryotes.

15 Catarino RR, Stark A. Assessing sufficiency and necessity of enhancer activities for gene expression and the mechanisms of transcription activation. Genes Dev. 32(3-4), 202-223 (2018).

16 Lewis MW, Li S, Franco HL, Lewis MW. Transcriptional control by enhancers and enhancer RNAs Transcriptional control by enhancers and enhancer RNAs. Transcription 00(4-5), 0-16 (2019).

17 Krimpenfort $\mathrm{P}$, de Jong $\mathrm{R}$, Uematsu $\mathrm{Y}$ et al. Transcription of T cell receptor beta-chain genes is controlled by a downstream regulatory element. EMBO J. 7(3), 745-750 (1988).

18 Bories JC, Demengeot J, Davidson L, Alt FW. Gene-targeted deletion and replacement mutations of the T-cell receptor $\beta$-chain enhancer: the role of enhancer elements in controlling V(D)J recombination accessibility. Proc. Natl Acad. Sci. USA 93(15), 7871-7876 (1996).

19 Bouvier G, Watrin F, Naspetti M, Verthuy C, Naquet P, Ferrier P. Deletion of the mouse T-cell receptor $\beta$ gene enhancer blocks $\alpha \beta$ T-cell development. Proc. Natl Acad. Sci. USA 93(15), 7877-7881 (1996).

20. Protocols.io.Strand specific detection of overlapping transcripts via purification involving denaturation of biotinylated cDNA. https://www.protocols.io/view/strand-specific-detection-o f-overlapping-transcrip-bfc5jiy6 
